\title{
Wireless positioning and path tracking for a mobile platform in greenhouse
}

\author{
Lijian $\mathrm{Yao}^{1}$, Dong Hu${ }^{1}$, Chao Zhao ${ }^{1}$, Zidong Yang ${ }^{1}$, Zhao Zhang ${ }^{2 *}$ \\ (1. School of Engineering, Zhejiang A\&F University, Hangzhou 311300, China; \\ 2. Department of Agricultural and Biosystems Engineering, North Dakota State University, Fargo, ND 58102, USA)
}

\begin{abstract}
In order to realize intelligent greenhouse, an automatic navigation method for a mobile platform based on ultra-wideband (UWB) positioning technology was proposed and validated in this study. The time difference of arrival (TDOA) approach was used to monitor and track the UWB positioning to obtain the localization information of the mobile platform working in a greenhouse. After applying polynomial fitting for positioning error correction, the system accuracy was within $5 \mathrm{~mm}$. A fuzzy controller model was constructed by incorporating the lateral and heading deviations as input variables and the steering angle of front wheel as the output variable. A fuzzy rule was established based on domain knowledge, as well as the steering angle of front wheel offline query table, which was applied to alleviate the calculative load of the controller. Experimental results confirmed that the automatic navigation method proposed in this study performed satisfactorily, with a steady-state error ranging from $41 \mathrm{~mm}$ to $79 \mathrm{~mm}$ when tracking straight line, and an average error of $185 \mathrm{~mm}$ and an average maximum error of $532 \mathrm{~mm}$ when tracking polygon. In addition, the maximum error occurred at the polygonal corner which could meet the needs of driving on the narrow road in the greenhouse. The method proposed in this study provides a new systematic approach for the research of greenhouse automatic navigation.
\end{abstract}

Keywords: wireless positioning, ultra-wideband, path tracking, positioning error correction, fuzzy control, query table, mobile platform, greenhouse

DOI: $10.25165 /$ j.ijabe. 20211401.5627

Citation: Yao L J, Hu D, Zhao C, Yang Z D, Zhang Z. Wireless positioning and path tracking for a mobile platform in greenhouse. Int J Agric \& Biol Eng, 2021; 14(1): 216-223.

\section{Introduction}

Greenhouse production can reduce the negative effect of extreme weather conditions, improve the efficiency of agricultural production, and lighten the labor intensity of farmers, thus, it gradually becomes a popular form of agricultural production. Intensive research focuses on the development of intelligent machinery suitable for greenhouse application, among which autonomous driving is one of the key topics. Precise positioning information, commonly obtained by using the global positioning system (GPS) technology, is critical for guiding autonomous agricultural machinery. Han et al. ${ }^{[1]}$ designed a path generation and tracking program for a mobile platform, which was installed on a laptop computer connecting to an RTK-GPS rover. With a sampling rate of $10 \mathrm{~Hz}$, the system could obtain average lateral errors from $3.8 \mathrm{~cm}$ to $12.8 \mathrm{~cm}$ on the straight path. An auto-guidance tillage tractor designed for a paddy application achieved an absolute position accuracy within $2 \mathrm{~cm}$ with a GNSS receiver installed for positioning ${ }^{[2]}$. Ye et al. ${ }^{[3]}$ designed a robotic

Received date: 2020-01-01 Accepted date: 2020-09-27

Biographies: Lijian Yao, Associate Professor, research interest: intelligent agricultural equipment and agricultural robot, Email: yao_njau@163.com; Dong Hu, Assistant Professor, research interest: information intelligent perception and quality nondestructive inspection of agricultural and forestry products, Email: hudong538338@zju.edu.cn; Chao Zhao, Associate Professor, research interest: perception and control of agricultural environment, Email: zhaochao@ zafu.edu.cn; Zidong Yang, Professor, research interest: design method of modern agricultural equipment, Email: 20150032@zafu.edu.cn.

*Corresponding author: Zhao Zhang, $\mathrm{PhD}$, Assistant Professor, research interest: automated agricultural machinery systems and smart agriculture. Department of Agricultural and Biosystems Engineering, North Dakota State University, Albrecht Blvd, Fargo, ND 58102, USA. Tel: +1-7012318403, Email: zhao.zhang.1@ndsu.edu. platform with a four-wheel-independent-steering system for bin management in orchard environment, consisting of a GPS receiver and an inertial measurement unit. Ortiz et al. ${ }^{[4]}$ employed an RTK-GPS-based auto-steer guidance system (accuracy ranging from 13-23 $\mathrm{mm}$ ) for evaluating the agronomic and economic benefits of peanut digging operations.

GPS signals could not be received accurately due to the shelter of building facilities for greenhouse application. Currently addressing the indoor positioning issues of vehicles has attracted the interest of academic and practical engineers. Schmidt et al ${ }^{[5]}$ used ultra-wideband (UWB) devices in industrial environments to reduce the need for relay nodes. Ahmed ${ }^{[6]}$ analyzed the advantages and problems of the time difference of arrival (TDOA) method of UWB positioning and presented two schemes (a positioning engine with dynamically changing anchor nodes and a time synchronization network) for improving the positioning accuracy. $\mathrm{Su}$ et al. $^{[7]}$ proposed a technique based on blind separation to achieve more accurate TDOA estimation of UWB positioning system. Yun et al. ${ }^{[8]}$ proposed a distance estimation method with an impulse radio ultra-wideband (IR-UWB) radar for a long-range target in the indoor environment, with an average error of less than $0.2 \mathrm{~m}$.

Path tracking is another important research topic in the field of unmanned driving. Pure pursuit model is a geometric path tracking method that is independent of vehicle model ${ }^{[9]}$. Based on the GPS signal, look-ahead distance was achieved for calculating the turning radius of an articulated tractor ${ }^{[10]}$. Look-ahead distance could affect the quality of path tracking in pure pursuit model. Repeated experiments or computer simulations are usually used to obtain the optimal look-ahead distance ${ }^{[11]}$. Park et al. ${ }^{[12]}$ proposed a method for dynamically modifying the look-ahead distance relative to a function of vehicle speed for vehicle path tracking. Ohta et al. ${ }^{[13]}$ employed the same method to determine 
the look-ahead distance by velocity of vehicle in urban road test. Fuzzy control can imitate the experience of experts or drivers, and it has been frequently used in automatic navigation. Abiyev et al. ${ }^{[14]}$ presented a design of fuzzy controller for a holonomic 4-wheel-driven soccer robots. To avoid obstacles efficiently and reach the target under many different shapes of obstacles, a fuzzy logic controller with 3 input variables and 2 output variables had been designed to improve the movement of the mobile robot ${ }^{[15]}$. Chen et al. ${ }^{[16]}$ designed a path tracking fuzzy logic controller for underwater vehicles, and the control rules were further optimized by a genetic algorithm. A hierarchically improved fuzzy controller was also proposed to solve the path tracking problem for the autonomous ground vehicle ${ }^{[17]}$. Although fuzzy control has the advantages of strong fault-tolerant ability and robustness, its real-time performance is limited and needs to be improved.

This study focused on the wheeled platform working in the greenhouse. The UWB chips were selected to build a wireless positioning system, coupled with the polynomial fitting method for correcting the positioning error. Based on the fuzzy control principle, the front wheel steering angle query table was constructed. The center of gravity was used to solve the fuzzy problem, so as to achieve the best steering angle output. Finally, the linear and rectangular path tracking experiments were carried out in the greenhouse to evaluate the effectiveness of the proposed method.

\section{Materials and methods}

\subsection{Test prototype}

The greenhouse mobile platform used in this study is pure electric 4-wheel driven, and is powered by a lithium battery ( $48 \mathrm{~V}$, 20 Ah; Lithium Battery, BD4820LK, Chilwee Group Co., LTD. Huzhou, Zhejiang, China). The length, width and height of the platform body were $1300 \mathrm{~mm} \times 700 \mathrm{~mm} \times 1250 \mathrm{~mm}$, and the tread and the wheelbase were $550 \mathrm{~mm}$ and $840 \mathrm{~mm}$, respectively. It could serve a variety of purposes in the greenhouse, such as spraying, fertilization, and transportation (Figure 1). The single-chip microcontroller STM32F767IGT6 (Xingyi Electronic Technology CO., Ltd, Guangzhou, China) was used as the main controller for the test of the platform. The wireless positioning system provided real-time position information $(x, y, z)$ for the main controller, and the transmission frequency of UWB module I-UWB LPS SA (SZ NoopLoop Technology Co., Ltd, Shenzhen, China) was 1-50 Hz. The electronic gyroscope WT901C (Wit-motion Shenzhen Co., LTD, Shenzhen, China) was used to provide heading angle information $\Theta$ for the main controller, and the measurement range was $-180^{\circ}-180^{\circ}$. The driver L298N (Hongchuang Microelectronics Technology Co., Ltd, Shenzhen, China) converted the Pulse Width Modulation (PWM) wave output by the main controller into voltage $U_{f}$, and transmitted it to the $24 \mathrm{~V}$ electric linear actuator (BXTL150, Louie Actuator Co., Ltd, Changzhou, Jiangsu, China) to push the trapezoid 4-bar mechanism to realize platform steering. The stroke was $200 \mathrm{~mm}$, and the rotation angle range of the front wheel was $-32^{\circ}-32^{\circ}$. The rear wheel motor driver (AQMD6020BLS, Aikong Electronics, Chengdu, China), which was driven by the direct current motor MK6012-G-400BN-T526 (YiJie Automation Co., Ltd, Shenzhen, China), converted the PWM wave into voltage $U_{r}$. The encoder fed back the stroke information of the front steering linear actuator and the rotation speed information of the rear wheel to the main controller in the form of Pulse Code Modulation (PCM) to realize closed-loop control. Additionally, the platform body was equipped with an ultrasonic sensor, remote controller, emergency stop button, warning light, and other safety auxiliary devices. The working principle of the control system is shown in Figure 2.

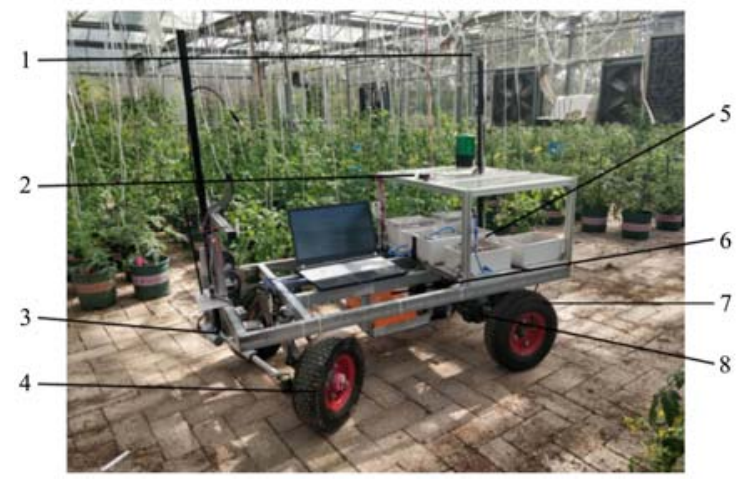

1. Mobile tag 2. Electronic gyroscope 3. Electric linear actuator 4. Front wheel 5. Main controller 6. Lithium battery 7.Rear wheel 8 . DC motor Figure 1 Greenhouse mobile platform prototype

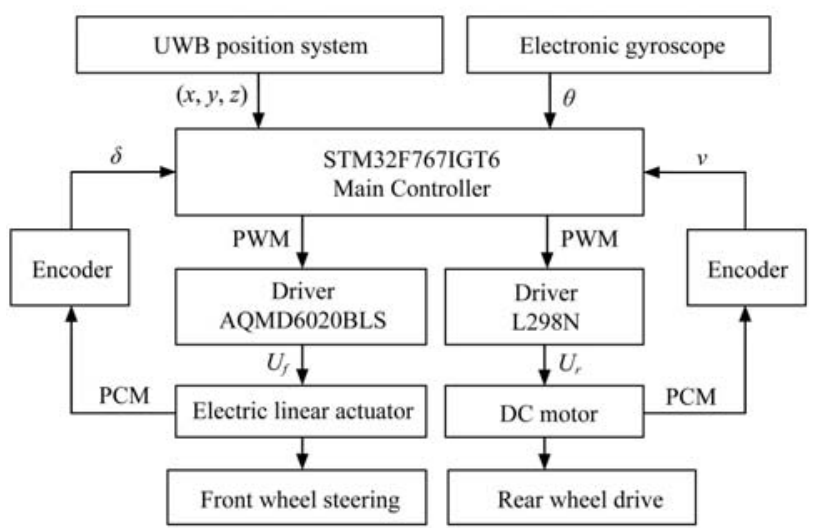

Note: $(x, y, z)$ is the position coordinates of mobile platform; $\theta$ is the heading angle information; $U_{f}$ and $U_{r}$ are the voltages; $\delta$ is the steering angle of the front wheels; $v$ is the moving speed of the platform

Figure 2 Working principle of the control system

\subsection{UWB positioning method}

\subsubsection{Positioning principle}

The mobile station (MS) with a tag was installed on the greenhouse platform. The real-time location information of the tag was provided by the UWB positioning system which was formed by 4 fixed base stations installed in the greenhouse. There were many UWB positioning algorithms ${ }^{[18]}$, among which the TDOA had the advantages of being independent on the synchronization of the tag with the base station clock and convenient networking. The TDOA method was also known as hyperbolic positioning ${ }^{[19]}$. By measuring the time difference between the UWB signal arriving at each positioning base station, the distance difference between the tag and each base station could be calculated ${ }^{[20]}$. A hyperbola was thus generated with the positioning base station as the focus and the distance difference as the long axis. The intersection of multiple sets of hyperbolae was the location of the mobile station, as shown in Figure 3. The calculation process of the algorithm included three steps.

Step1: The position coordinate of the mobile station was $\left(x_{0}\right.$, $y_{0}$ ), and the position coordinates of the four positioning base stations $\mathrm{BS}_{1}-\mathrm{BS}_{4}$ were $\left(x_{i}, y_{i}\right), i=1,2,3,4$. Since the experiments in this study were conducted in a relatively flat greenhouse, the vertical coordinate of MS was not taken into account. The distance $R_{i}(\mathrm{~m})$ between the MS and each base station was:

$$
R_{i}=\sqrt{\left(x_{0}-x_{i}\right)^{2}+\left(y_{0}-y_{i}\right)^{2}}
$$




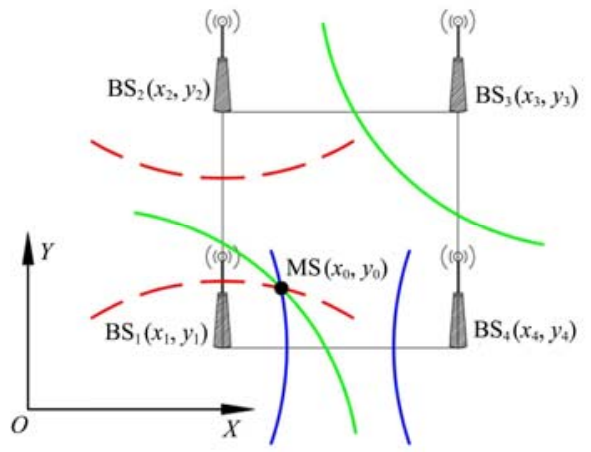

Note: BS is the base station; MS is the mobile station.

Figure 3 Positioning principle of TDOA

Step2: Taking the base station pairs $\left(\mathrm{BS}_{1}, \mathrm{BS}_{2}\right),\left(\mathrm{BS}_{1}, \mathrm{BS}_{3}\right)$, and $\left(\mathrm{BS}_{1}, \mathrm{BS}_{4}\right)$ as the focal points of the hyperbola respectively, the distance difference that the signal sent from MS traveled to the station pairs were constant, so that three sets of hyperbolae could be obtained. Then the $d_{j, 1}(\mathrm{~m})$ is the difference in the distance between $R_{j}$ and $R_{1}$ is

$$
d_{j, 1}=R_{j}-R_{1}=c \times \tau_{j, 1}
$$

where, $\tau_{j, 1}$ is the transmission time difference between the base station $j$ to the MS and the first base station to the MS, $\mathrm{m} ; j=2,3,4$; $c$ is the speed of light, $3 \times 10^{8} \mathrm{~m} / \mathrm{s}$.

Step3: Since the coordinates of the four base stations were known, and the signal transmission time between each base station and the MS could be obtained by the UWB chips, the coordinate of the MS could be calculated by Chan algorithm ${ }^{[21]}$. Therefore, the calculation equation of coordinate $\left(x_{0}, y_{0}\right)$ was as follows:

$$
\boldsymbol{G} \mathbf{z}^{0}=\boldsymbol{h}
$$

where,

$$
\mathbf{z}^{0}=\left[\begin{array}{l}
x_{0} \\
y_{0} \\
R_{1}
\end{array}\right] ; \quad \boldsymbol{h}=\frac{1}{2}\left[\begin{array}{l}
d_{2,1}^{2}-K_{2}+K_{1} \\
d_{3,1}^{2}-K_{3}+K_{1} \\
d_{4,1}^{2}-K_{4}+K_{1}
\end{array}\right] ; \quad \boldsymbol{G}=-\left[\begin{array}{lll}
x_{2,1} & y_{2,1} & d_{2,1} \\
x_{3,1} & y_{3,1} & d_{3,1} \\
x_{4,1} & y_{4,1} & d_{4,1}
\end{array}\right] ;
$$

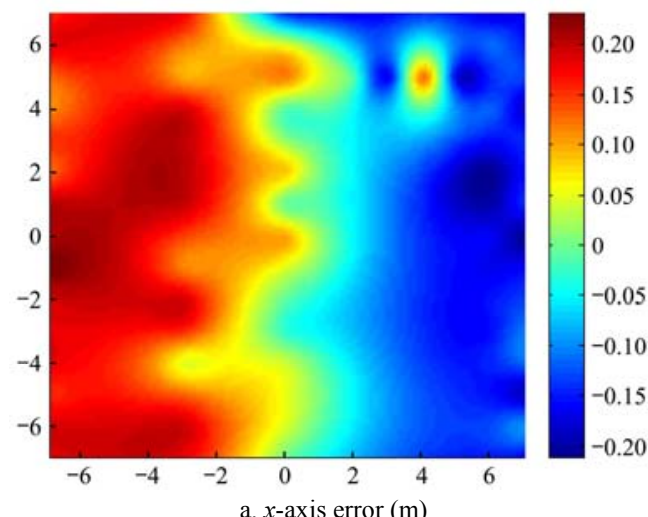

$$
K_{i}=x_{i}^{2}+y_{i}^{2}, i=1,2 \ldots \ldots .4 ; x_{i, 1}=x_{i}-x_{1}, i=1,2,3,4 .
$$

\subsubsection{Analysis of positioning errors}

Positioning was prone to error due to the reflection of UWB signals by the walls, columns, and cultivating beds in the greenhouse. This error was difficult to explain with accurate formula and theory. In this study, an error model was established by equation fitting to correct the UWB positioning error.

An area of $15 \mathrm{~m} \times 15 \mathrm{~m}$ was selected in the greenhouse where the UWB positioning system was installed, and a total of 256 intersections of $1 \mathrm{~m} \times 1 \mathrm{~m}$ were drawn (figure 4). The standard coordinates $\left(\left(x_{k}, y_{k}\right), k=1,2, \cdots, 256\right)$ of these 256 intersections in the $X O Y$ coordinate system were recorded. Placing the MS on these 256 intersections in turn, the method in section 2.2.1 was used to calculate the measured coordinate of the MS, which was recorded as $\left(x_{l}, y_{l}\right), l=1,2, \cdots, 256$.

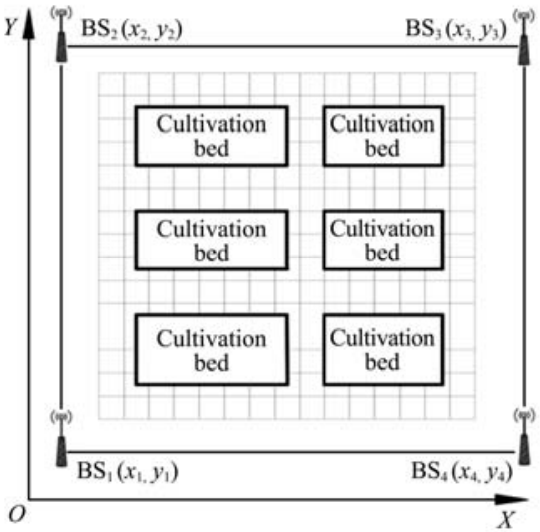

Figure 4 Schematic diagram of positioning test

The measured values of each intersection were compared with the standard coordinate values, the difference between the horizontal and vertical coordinates $(\Delta Z x, \Delta Z y)$ could be found. A three-dimensional error elevation map was constructed in the $\Delta Z x-X-Y$ and $\Delta Z y-X-Y$ spaces, as shown in Figure 5.

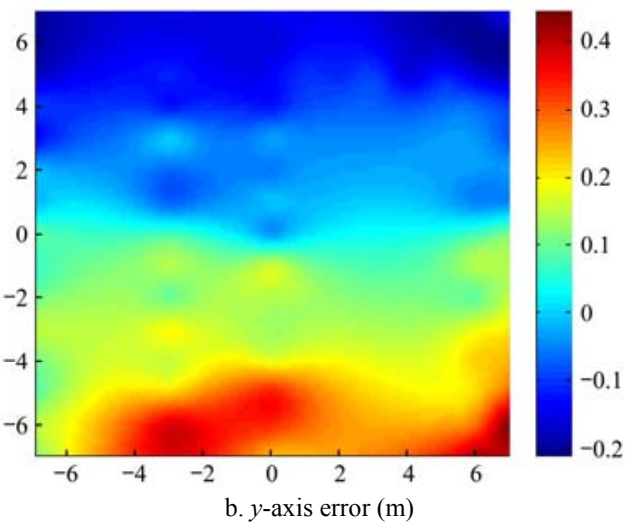

Note: $x$-axis and $y$-axis represent the direction of width and length of the greenhouse, respectively, m. Considering the convenience of calculation, the coordinate center is at the center of the greenhouse.

Figure 5 Positioning error elevation maps

\subsubsection{Correction of positioning errors}

In order to find the regularity of the positioning error, a two-variable polynomial surface was fitted to describe the error distribution. It was found that the higher the order was, the higher the precision was, but high order resulted in a greater amount of calculation. The method for determining the polynomial order was implemented in several steps. First, the polynomial order $t$ was set, and the residual sum of squares $S_{t}$ was calculated after the establishment of the model. Then the fitting model was built with $t-1$ order, and the residual sum of squares $S_{t-1}$ was used as the $F$ distribution statistic. The parameter $F_{\alpha}$ was obtained by checking $F$ distribution according to the significant level $\alpha$, and the degree of freedom. If $F<F_{a}$, the order of the polynomial was $t-1$, otherwise it was $t$. In this study, a 4-order model with 15 coefficients was chosen based on the preliminary experiments. The fitting was conducted with MATLAB ${ }^{\circledR}$ 2016a. The fitted error model according to Figure 5 was shown in Equation (5), with the coefficients listed in Table 1 .

$$
\begin{aligned}
& z(x, y)=m_{4} x^{4}+m_{3} x^{3}+m_{2} x^{2}+m_{1} x++n_{4} y^{4}+n_{3} y^{3}+n_{2} y^{2}+n_{1} y+p_{31} x^{3} y+ \\
& p_{13} x y^{3}+p_{22} x^{2} y^{2}+p_{21} x^{2} y+p_{12} x y^{2}+p_{11} x y+p_{0}
\end{aligned}
$$


where, $z(x, y)$ is the bivariate polynomial fitting equation of error; $x$ and $y$ represent the horizontal and vertical coordinates of the measuring point, respectively.

Table 1 Coefficients of error model

\begin{tabular}{ccccc}
\hline Coefficients & $m_{4}$ & $m_{3}$ & $m_{2}$ & $m_{1}$ \\
\hline$Z_{X}(x, y)$ & $-3.52 \mathrm{e}-06$ & $4.10 \mathrm{e}-04$ & $5.60 \mathrm{e}-04$ & $-4.40 \mathrm{e}-02$ \\
$Z_{Y}(x, y)$ & $4.71 \mathrm{e}-06$ & $2.00 \mathrm{e}-04$ & $6.33 \mathrm{e}-05$ & $-8.30 \mathrm{e}-03$ \\
\hline Coefficients & $n_{4}$ & $n_{3}$ & $n_{2}$ & $n_{1}$ \\
\hline$Z_{X}(x, y)$ & $-2.38 \mathrm{e}-06$ & $-1.10 \mathrm{e}-04$ & $5.00 \mathrm{e}-04$ & $4.60 \mathrm{e}-03$ \\
$Z_{Y}(x, y)$ & $2.21 \mathrm{e}-06$ & $1.60 \mathrm{e}-04$ & $4.00 \mathrm{e}-04$ & $-4.10 \mathrm{e}-02$ \\
\hline \hline Coefficients & $p_{31}$ & $p_{13}$ & $p_{22}$ & $p_{21}$ \\
\hline$Z_{X}(x, y)$ & $-2.25 \mathrm{e}-06$ & $1.22 \mathrm{e}-06$ & $2.27 \mathrm{e}-06$ & $-6.74 \mathrm{e}-05$ \\
$Z_{Y}(x, y)$ & $-4.38 \mathrm{e}-06$ & $-8.28 \mathrm{e}-06$ & $-4.55 \mathrm{e}-06$ & $-5.32 \mathrm{e}-06$ \\
\hline \hline Coefficients & $p_{12}$ & $p_{11}$ & $p_{0}$ & \\
\hline$Z_{X}(x, y)$ & $5.22 \mathrm{e}-06$ & $6.00 \mathrm{e}-04$ & $3.30 \mathrm{e}-02$ & \\
$Z_{Y}(x, y)$ & $1.80 \mathrm{e}-04$ & $1.70 \mathrm{e}-03$ & $4.00 \mathrm{e}-02$ & \\
\hline
\end{tabular}

It could be observed from Equation (5) and Table 1 that the coefficients of the 4th term of $x$ and $y$ in the $X$-direction were $-3.52 \mathrm{e}-06$ and $-2.38 \mathrm{e}-06$, respectively, and the maximum correction ability for errors was $-0.0085 \mathrm{~m}\left(-3.52 \mathrm{e}-06 \times 7^{4}\right)$ and $-0.0057 \mathrm{~m}\left(-2.38 \mathrm{e}-06 \times 7^{4}\right)$, which was less than $1 \mathrm{~cm}$. Therefore, the errors could be ignored, and the error model was simplified as:

$$
z(x, y)=m_{3} x^{3}+m_{2} x^{2}+m_{1} x+n_{3} y^{3}+n_{2} y^{2}+n_{1} y+p_{12} x y^{2}+p_{11} x y+p_{0}
$$

$$
\begin{aligned}
& \mathrm{Z}_{X}(x, y)=0.00041 x^{3}+0.00056 x^{2}-0.0439 x-0.00011 y^{3}+ \\
& 0.0005 y^{2}+0.0046 y-0.000067 x^{2} y+0.0006 x y+0.0335 \\
& \mathrm{Z}_{Y}(x, y)=0.0002 x^{3}+0.000063 x^{2}-0.0083 y+0.00016 y^{3} 0.0004 y^{2}- \\
& 0.041 y+0.00018 x y^{2}+0.0017 x y+0.0397
\end{aligned}
$$

where, $Z x(x, y)$ is error model on $x$-axis; $Z y(x, y)$ is error model on $y$-axis.

Statistically, the closer the determination coefficient $R^{2}$ is to 1 , the better the fitting is. The determination coefficients of the two fitting equations used in this study were 0.912 and 0.947 , respectively, indicating that the fitness of the models was good. Hence, the positioning correction equation is

$$
\left\{\begin{array}{l}
x_{\text {correction }}=x+Z_{X}(x, y) \\
y_{\text {correction }}=y+Z_{Y}(x, y)
\end{array}\right.
$$

where, $x_{\text {correction }}$ and $y_{\text {correction }}$ were horizontal and vertical coordinates of the measurement points after correction, respectively.

In order to evaluate the performance of the error correction equation, the measured coordinates of 256 points $(x, y)$ were put into Equations (7), (8) and (9). The corrected errors are shown in Figure 6 . The average errors before and after the correction were calculated. The results are shown in Table 2.

According to Table 2, the average errors of $x$-axis and $y$-axis directions were reduced by $0.09 \mathrm{~m}$ and $0.12 \mathrm{~m}$, respectively. The improvements in positioning accuracy were $69.23 \%$ and $63.16 \%$, demonstrating that the error correction equation could significantly improve the positioning accuracy of UWB.

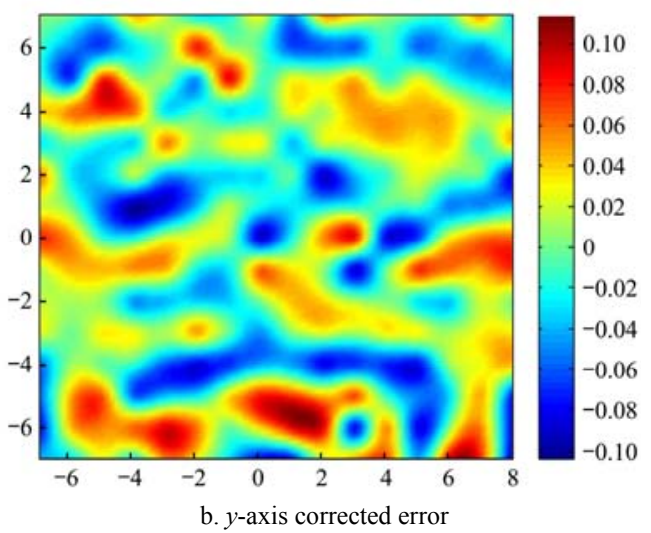

Note: $x$-axis and $y$-axis represent the direction of width and length of the greenhouse, respectively, m. Considering the convenience of calculation, the coordinate center is at the center of the greenhouse.

Figure 6 Corrected positioning error elevation maps

Table 2 Average error before and after the correction

\begin{tabular}{cccc}
\hline $\begin{array}{c}\text { Error } \\
\text { direction }\end{array}$ & $\begin{array}{c}\text { Before } \\
\text { correction } / \mathrm{m}\end{array}$ & $\begin{array}{c}\text { After } \\
\text { correction } / \mathrm{m}\end{array}$ & $\begin{array}{c}\text { Rate of positioning } \\
\text { improvement } \%\end{array}$ \\
\hline$x$-axis & 0.13 & 0.04 & 69.23 \\
$y$-axis & 0.19 & 0.07 & 63.16 \\
\hline
\end{tabular}

\subsection{Design of fuzzy controller}

2.3.1 Input and output variables

The input variables of the fuzzy controller were the lateral deviation $d(\mathrm{~m})$ and heading deviation $\theta\left(^{\circ}\right)$ of the platform relative to the desired path, and the domain were $[-0.6,0.6]$ and $\left[-60^{\circ}, 60^{\circ}\right]$ respectively, which were determined by the width of greenhouse road and the mechanical structure of the platform. The output variable was the steering angle of front wheel $\delta$ of the platform, and the discussion range was $\left[-32^{\circ}, 32^{\circ}\right]$. The sign of each variable was defined as follows: when the platform was located on the left side of the desired path, the lateral deviation was positive; otherwise, it was negative. When the heading of the platform was on the left side of the desired path or the front wheel turned left, $\theta$ and $\delta$ were positive; otherwise, they were negative. Considering the accuracy and flexibility of the control system, the input and output variables were categorized into 5 fuzzy subsets (NB, NS ZO, $\mathrm{PS}, \mathrm{PB})$. When the platform deviation was large, the large-scale fuzzy subsets could be used to obtain a better rate of convergence. When the deviation was small, which meant the platform was approaching the desired path, the high-resolution (small-scale) fuzzy subsets could be used to achieve more precise control accuracy of position and attitude of the platform. The triangle membership function was used for each input and output variable (Figure 7).

2.3.2 Rules for fuzzy controller

According to the domain knowledge, the following principles were followed when designing control rules: when the lateral and heading deviations were large, a large $\delta$ should be used to drive the platform to converge to the desired path quickly; when the deviation was small, a small $\delta$ of fine adjustment should be adopted to ensure the stability of the platform. Based on the above principles and repeated attempts for optimization, 25 control rules were designed, as shown in Table 3. 

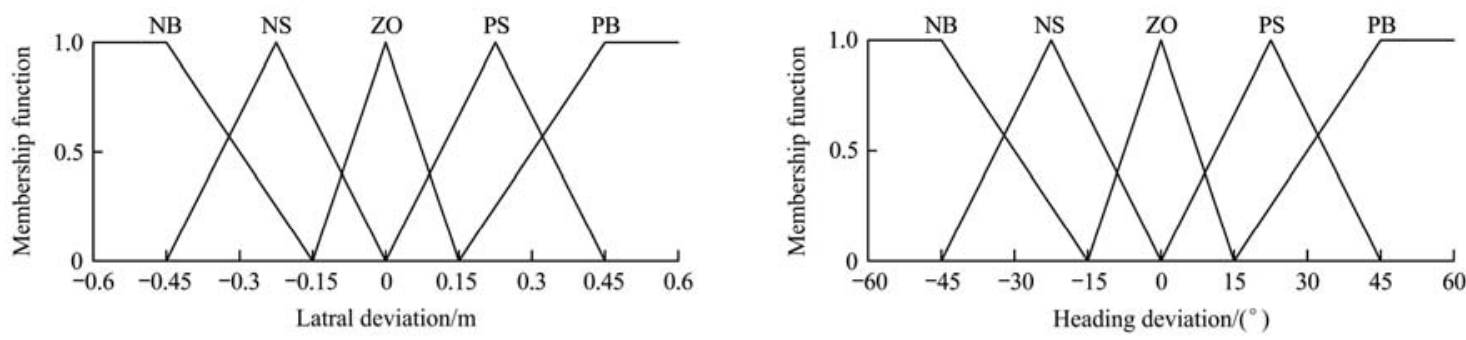

a. Lateral deviation $d(\mathrm{~m})$

b. Heading deviation $\theta\left(^{\circ}\right)$

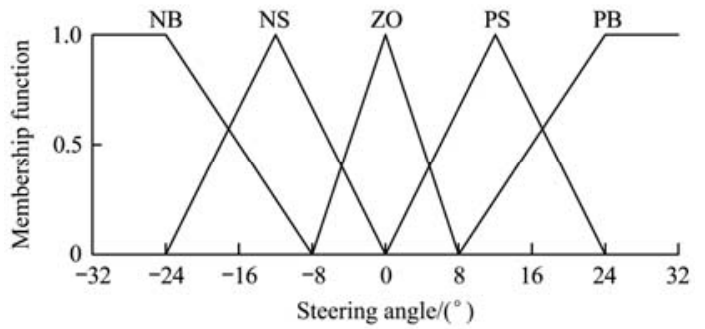

c. Steering angle of front wheel $\delta\left(^{\circ}\right)$

Note: N, P, B, M, S and ZO denote negative, positive, big, middle, small, and zero, respectively.

Figure 7 Membership functions for each input and output variable

Table 3 Rules for fuzzy controller

\begin{tabular}{|c|c|c|c|c|c|c|}
\hline \multicolumn{2}{|c|}{$\delta /\left(^{\circ}\right)$} & \multicolumn{5}{|c|}{$\theta /\left(^{\circ}\right)$} \\
\hline & & NB & NS & $\mathrm{ZO}$ & PS & PB \\
\hline \multirow{4}{*}{$d / \mathrm{m}$} & NB & $P B$ & $P B$ & $P B$ & $P S$ & $Z O$ \\
\hline & NS & $P B$ & $P B$ & $P S$ & $Z O$ & $N S$ \\
\hline & $\mathrm{ZO}$ & $P B$ & $P S$ & $Z O$ & $N S$ & $N B$ \\
\hline & PS & $P S$ & $Z O$ & $N S$ & $N B$ & $N B$ \\
\hline
\end{tabular}

Note: N, P, B, M, S and ZO denote negative, positive, big, middle, small, and zero, respectively.

\subsubsection{Designing Query table}

In order to reduce the on-line operation of the controller, the two input variables were subdivided into 13 values to calculate the steering angle, thus a query table was formed (Table 4). In actual control, the only need is to query this control table. The fuzzy control method of off-line calculating and on-line querying table could meet the requirements of fast response and real-time control of the system.

Table 4 Fuzzy control query table of steering angle of front wheel

\begin{tabular}{|c|c|c|c|c|c|c|c|c|c|c|c|c|c|c|}
\hline \multirow{2}{*}{\multicolumn{2}{|c|}{$\delta /\left({ }^{\circ}\right)$}} & \multicolumn{13}{|c|}{$\theta /\left({ }^{\circ}\right)$} \\
\hline & & -60 & -45 & -30 & -20 & -10 & -5 & 0 & 10 & 20 & 30 & 45 & 60 & 10 \\
\hline \multirow{13}{*}{$d / \mathrm{m}$} & -0.6 & 32 & 32 & 32 & 32 & 32 & 32 & 30 & 26 & 21 & 16 & 11 & 0 & 0 \\
\hline & -0.45 & 32 & 32 & 32 & 32 & 31 & 30 & 28 & 26 & 21 & 16 & 11 & 0 & 0 \\
\hline & -0.3 & 32 & 32 & 32 & 32 & 23 & 22 & 22 & 17 & 11 & 5 & 0 & -11 & -11 \\
\hline & -0.2 & 32 & 32 & 32 & 30 & 23 & 21 & 20 & 15 & 9 & 0 & -5 & -16 & -16 \\
\hline & -0.1 & 32 & 32 & 23 & 23 & 13 & 12 & 11 & 4 & 0 & -9 & -11 & -21 & -21 \\
\hline & -0.05 & 31 & 30 & 22 & 21 & 12 & 6 & 6 & 0 & -4 & -15 & -17 & -26 & -26 \\
\hline & 0 & 29 & 28 & 22 & 20 & 11 & 6 & 0 & -6 & -11 & -20 & -22 & -28 & -29 \\
\hline & 0.05 & 26 & 26 & 17 & 15 & 4 & 0 & -6 & -6 & -12 & -21 & -22 & -30 & -31 \\
\hline & 0.1 & 21 & 21 & 11 & 9 & 0 & -4 & -11 & -12 & -13 & -23 & -23 & -32 & -32 \\
\hline & 0.2 & 16 & 16 & 5 & 0 & -9 & -15 & -20 & -21 & -23 & -30 & -32 & -32 & -32 \\
\hline & 0.3 & 11 & 11 & 0 & -5 & -11 & -17 & -22 & -22 & -23 & -32 & -32 & -32 & -32 \\
\hline & 0.45 & 0 & 0 & -11 & -16 & -21 & -26 & -28 & -30 & -31 & -32 & -32 & -32 & -32 \\
\hline & 0.6 & 0 & 0 & -11 & -16 & -21 & -26 & -30 & -32 & -32 & -32 & -32 & -32 & -32 \\
\hline
\end{tabular}

\subsubsection{Defuzzification of output variables}

When any pair of lateral deviation $d$ and heading deviation $\theta$ were input, its output value $\delta$ must be in the area enclosed by 4 adjacent steering angles, as shown in Table 5. Under this situation, the output steering angle of front wheel $\delta$ could be clarified by calculating the center of gravity.

Then, when $\theta$ and $d$ are between $\theta_{\sigma+1}, \theta_{\sigma}$ and $d_{\tau+1}, d_{\tau}$ respectively, the output steering angle is

$$
\delta=\left(\delta_{1}+\delta_{2}+\delta_{3}+\delta_{4}\right) / 4
$$

where, $\delta_{1}, \delta_{2}, \delta_{3}$ and $\delta_{4}$ can be expressed as:

$$
\left\{\begin{array}{l}
\delta_{1}=\delta_{\tau, \sigma}\left[1+\left(\theta-\theta_{\sigma}\right) /\left(\theta_{\sigma+1}-\theta_{\sigma}\right)\right] \\
\delta_{2}=\delta_{\tau+1, \sigma}\left[1+\left(\theta-\theta_{\sigma}\right) /\left(\theta_{\sigma+1}-\theta_{\sigma}\right)\right] \\
\delta_{3}=\delta_{\tau, \sigma}\left[1+\left(d-d_{\tau}\right) /\left(d_{\tau+1}-d_{\tau}\right)\right] \\
\delta_{4}=\delta_{\tau+1, \sigma}\left[1+\left(d-d_{\tau}\right) /\left(d_{\tau+1}-d_{\tau}\right)\right]
\end{array}\right.
$$


Table 5 Part of fuzzy control query table

\begin{tabular}{ccc}
\hline$\delta$ & $\theta_{\sigma}$ & $\theta_{\sigma+1}$ \\
\hline$d_{\tau}$ & $\delta_{\tau, \sigma}$ & $\delta_{\tau, \sigma+1}$ \\
$d_{\tau+1}$ & $\delta_{\tau+1, \sigma}$ & $\delta_{\tau+1, \sigma+1}$ \\
\hline
\end{tabular}

Note: $\theta_{\sigma+1}$ and $\theta_{\sigma}$ are the upper and lower limits of the current heading deviation shown in Table 4. $d_{\tau+1}$ and $d_{\tau}$ are the upper and lower limits of the current lateral deviation shown in Table 4

\section{Results and discussion}

\subsection{Testing process}

In order to evaluate the effectiveness of the proposed algorithm, path tracking tests were conducted in Guantang Farm greenhouse of Zhejiang Agricultural and Forestry University from June to August in 2019. The tests were divided into two types, namely, linear path tracking and polygonal path tracking. Small tomatoes and peppers were mainly planted in the greenhouse with the size of $25 \mathrm{~m} \times 18 \mathrm{~m} \times 3 \mathrm{~m}$. There was a road of $1.5 \mathrm{~m}$ width between each cultivation bed for operating the platform. The height of each UWB base station and the MS from the ground was $1.6 \mathrm{~m}$, and the projection point of the MS was located in the middle of the rear axle of the test platform. The sampling frequency was $5 \mathrm{~Hz}$. The platform attitude information was obtained by the gyroscope installed on the platform body. The platform speed was $0.5 \mathrm{~m} / \mathrm{s}$, and the upper computer continuously recorded the position information of the platform during the test.

Average error, steady-state error and stable distance were selected to evaluate the performance of the path tracking method. The average error represents the mean value of all lateral deviations from the beginning to the end of the test. The stable distance refers to the first convergence of the platform from the maximum deviation state to the lateral deviation within $\pm 0.1 \mathrm{~m}$. It is the horizontal distance that the platform passes when reaching a stable state. The steady-state error refers to the mean value of the lateral deviation after the stable state. Average error and steady-state error are important indexes to reflect the accuracy of path tracking. The stable distance reflects the speed of the platform from a large deviation to a small deviation in path tracking.

\subsubsection{Linear tracking test}

The main road in the middle of the planting frame was used as the expected path of linear tracking. According to the deviation between the platform and the expected path, four different initial states of $(d, \theta)$ were designed to investigate the performance of the straight-line path tracking, which were $\left(0.5 \mathrm{~m},-55^{\circ}\right),(-0.55 \mathrm{~m}$, $\left.15^{\circ}\right),\left(0.13 \mathrm{~m},-30^{\circ}\right)$ and $\left(-0.1 \mathrm{~m}, 60^{\circ}\right)$. The track curves of the four initial state straight line tracking tests were shown in Figure 8, and the data statistics were shown in Table 6.
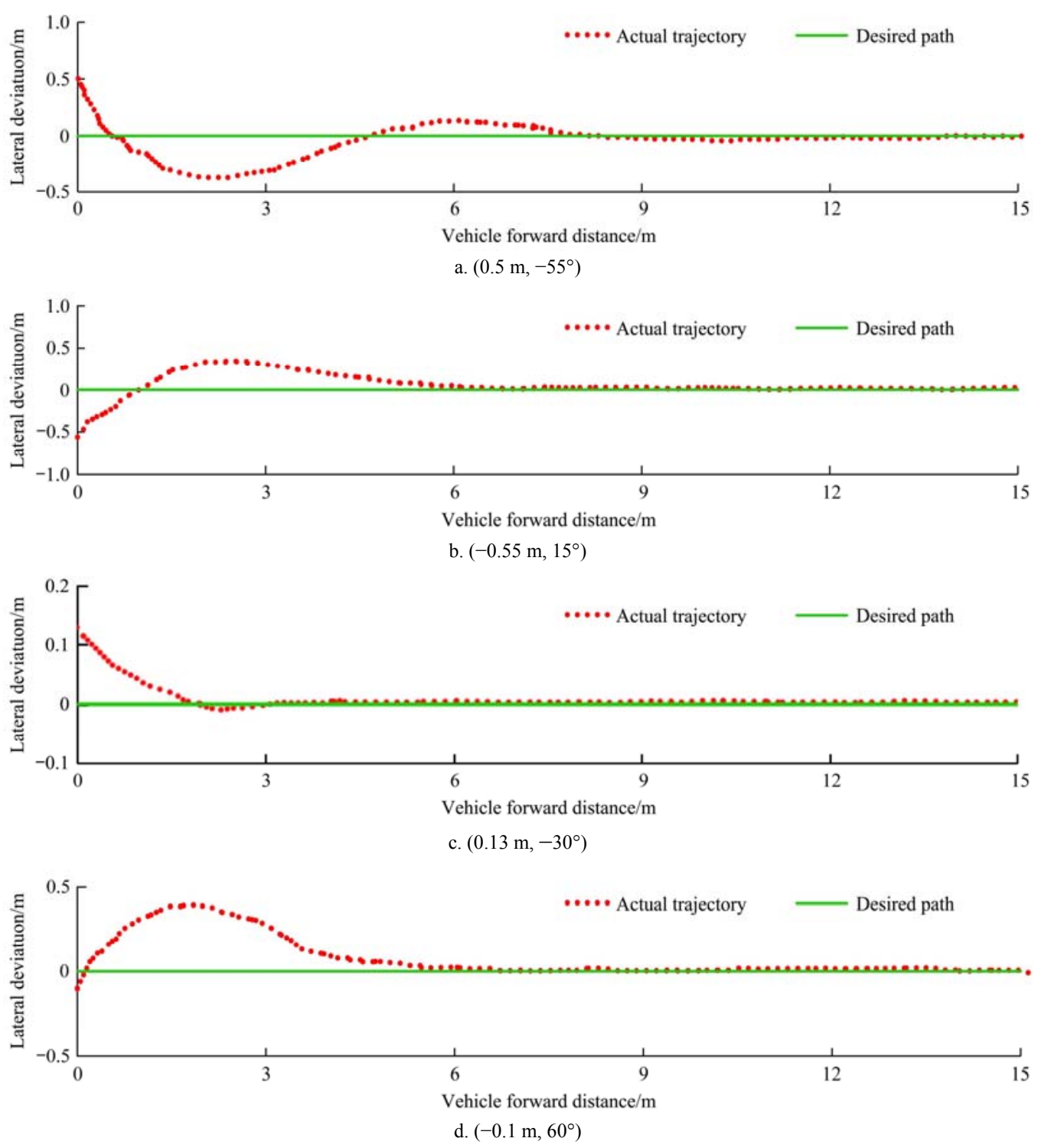

Figure 8 Linear path tracking test performance under four different initial states 
Table 6 Statistics of linear path tracking test

\begin{tabular}{ccccc}
\hline $\begin{array}{c}\text { Initial states } \\
\left(d / \mathrm{m}, \theta /\left(^{\circ}\right)\right)\end{array}$ & $\left(0.5,-55^{\circ}\right)$ & $\left(-0.55,5^{\circ}\right)$ & $\left(0.13,-30^{\circ}\right)$ & $\left(-0.1,60^{\circ}\right)$ \\
\hline Average error/mm & 109 & 117 & 58 & 86 \\
Steady-state error/mm & 79 & 68 & 41 & 76 \\
Stable distance $/ \mathrm{mm}$ & 4105 & 5148 & 2395 & 4031 \\
\hline
\end{tabular}

3.1.2 Polygonal path tracking

As shown in Figure 9, the platform started from the coordinate $(8,0)$ moved toward the positive direction of $Y$-axis, passed through points $(8,5),(14,5),(14,14),(1,14)$ and $(1,0)$ respectively, and then went back to the initial starting point, completing the tracking test of the whole closed polygonal path. Four tests were carried out, and the statistical results of test data were shown in Table 7.

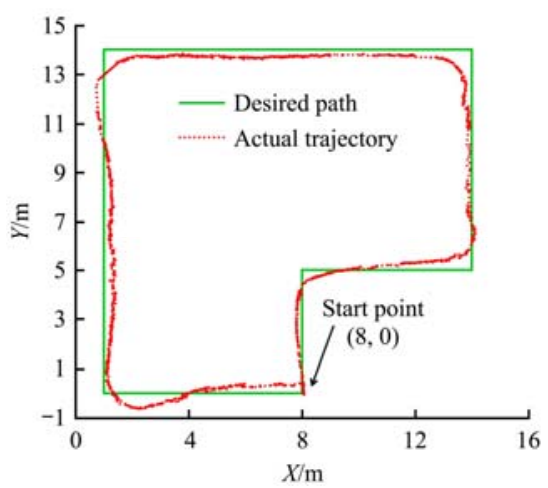

Figure 9 Polygonal path tracking test

Table 7 Statistics of polygonal path tracking test

\begin{tabular}{ccc}
\hline Tests number & Average error/mm & Maximum error/m \\
\hline 1 & 213 & 610 \\
2 & 173 & 472 \\
3 & 197 & 584 \\
4 & 155 & 462 \\
Average of 4 tests & 185 & 532 \\
\hline
\end{tabular}

\subsection{Analysis and discussion}

(1) In the straight path tracking, the average errors of four different initial states were $109 \mathrm{~mm}, 117 \mathrm{~mm}, 58 \mathrm{~mm}$ and $86 \mathrm{~mm}$, respectively, as shown in Table 6 . When the platform reached a stable state, the steady-state errors were $79 \mathrm{~mm}, 68 \mathrm{~mm}, 41 \mathrm{~mm}$ and $76 \mathrm{~mm}$, all of which were less than $100 \mathrm{~mm}$. In the polygonal path tracking, the average errors of the four tests were $213 \mathrm{~mm}$, $173 \mathrm{~mm}, 197 \mathrm{~mm}$, and $155 \mathrm{~mm}$, with a comprehensive average error of $185 \mathrm{~mm}$. Compared with the road of $1.5 \mathrm{~m}$ width between the cultivation beds, the proportion of this error is only $12.3 \%$. The results showed that when the platform was driving in the greenhouse, it had a high path tracking accuracy. The error of polygonal driving was higher than that of straight-line driving because polygonal tracking needed to switch to new tracking paths frequently.

(2) In the linear tracking test, the distances from initial to stable state under 4 different initial states were $4105 \mathrm{~mm}, 5148 \mathrm{~mm}$, $2395 \mathrm{~mm}$ and $4031 \mathrm{~mm}$, respectively, which were caused by the inconsistency of their initial states. If the initial deviation was large (as shown in Figure 8), the stable distance was large, and the maximum deviation was large; otherwise, if the initial deviation was small (as shown in Figure 8c), the stable distance was short, and the maximum deviation was small correspondingly.

(3) In the polygonal path tracking, the maximum deviation was $610 \mathrm{~mm}, 472 \mathrm{~mm}, 584 \mathrm{~mm}$ and $462 \mathrm{~mm}$, with an average maximum deviation of $532 \mathrm{~mm}$. It was found that the maximum deviation occurred at the $90^{\circ}$ turn, which was caused by the change of the current tracking path.

\section{Conclusions}

(1) During construction and testing of the platform system in the greenhouse, UWB module was adopted to build the positioning system, TDOA method was used to obtain the location information of the working platform, and the polynomial model was applied to correct the errors, resulting in a decrease of the average error in the direction of horizontal and vertical coordinates by $0.09 \mathrm{~m}$ and $0.12 \mathrm{~m}$ respectively, and an improvement for the positioning accuracy by $69.23 \%$ and $63.16 \%$. It showed that the error correction equation could significantly improve the accuracy of UWB positioning system.

(2) The input variables of the fuzzy controller were the lateral deviation $d$ and heading deviation $\theta$ between the platform and the desired path, and the output variable was the steering angle of front wheel $\delta$ of the platform. The fuzzy controller was constructed according to the experience of experts and drivers. According to the deviation of input variables, the uneven quantization level was adopted taking into account the control accuracy of the system and the flexibility of optimization. The steering angle of front wheel query table was also designed to reduce the online calculative burden of the controller.

(3) The path tracking tests of straight line and polygon were carried out in greenhouse. When the platform was tracking under 4 initial states, the steady-state deviation was between $41-79 \mathrm{~mm}$, and the average error of the polygonal path was $185 \mathrm{~mm}$, which could meet the needs of driving on the narrow road in the greenhouse.

(4) It was observed in the experiment that the change of temperature and shelter in greenhouse would affect the positioning accuracy. In the process of path tracking, the wheel may sideslip, and this could significantly affect the accuracy of path tracking. In the future, it is necessary to continuously improve the mechanical performance of the platform, and test the positioning accuracy of UWB in different environments to further improve the quality of path tracking in greenhouse.

\section{Acknowledgements}

This work was financially supported by the Zhejiang Science and Technology Department Basic Public Welfare Research Project (Grant No. LGN18F030001) and the Major Project of Zhejiang Science and Technology Department (Grant No. 2016C02G2100540).

\section{[References]}

[1] Han X, Kim H, Moon H, Woo H, Kim J, Kim Y. Development of a path generation and tracking algorithm for a Korean auto-guidance tillage tractor. Journal of Biosystems Engineering, 2013; 38(1): 1-8.

[2] Han X, Kim H, Kim J, Yi S, Moon H, Kim J, et al. Path-tracking simulation and field tests for an auto-guidance tillage tractor for a paddy field. Computers and Electronics in Agriculture, 2015; 112: 161-171.

[3] Ye Y, He L, Zhang Q. A robotic platform "Bin-Dog" for bin management in orchard environment. 2016 ASABE Annual International Meeting, Orlando, USA: ASABE, 2016; pp.1-8.

[4] Ortiz B V, Balkcom K B, Duzy L, Santen E, Hartzog D L. Evaluation of agronomic and economic benefits of using RTK-GPS-based auto-steer guidance systems for peanut digging operations. Precision Agriculture, 2013; 14(4): 357-375.

[5] Schmidt J F, Neuhold D, Klaue J, Schupkex D, Bettstetter C. Experimental study of UWB connectivity in industrial environment. 24th 
European Wireless Conference, Catania, Italy: VDE, 2018; pp.1-4.

[6] Ahmed S N A, Zeng Y. UWB positioning accuracy and enhancements. 2017 IEEE Region 10 Conference (TENCON), Penang, Malaysia: IEEE, 2017; pp.634-638.

[7] Su T, Gao Y. TDOA estimation of dual-satellites interference localization based on blind separation. Journal of Systems Engineering and Electronics, 2019; 30(4): 696-702.

[8] Yun Y, Park Y, Lee B M, Hyun B, Kim Y. Distance estimation scheme exploiting IR-UWB radar with clutter suppressing algorithm in indoor environments. Journal of Electrical Engineering \& Technology, 2019(14) 1759-1769.

[9] Amidi O. Integrated mobile robot control. International Society for Optics and Photonics, 1990; 91:504-523.

[10] Rains G C, Faircloth A G, Thai C, Raper R L. Evaluation of a simple pure pursuit path-following algorithm for an autonomous, articulated-steer vehicle. Applied Engineering in Agriculture, 2014; 30(3): 367-374.

[11] Ye Y, He L, Zhang Q. Steering control strategies for a four-wheel-independent-steering bin managing robot. International Federation of Automatic Control, 2016; 49(16): 39-44.

[12] Park M W, Lee S W, Han W Y. Development of lateral control system for autonomous vehicle based on adaptive pure pursuit algorithm. 2014 14th International Conference on Control, Automation and Systems (ICCAS 2014), Seoul, Korea: IEEE, 2014; pp.1443-1447.

[13] Ohta H, Akai N, Takeuchi E, Kato S, Edahiro M. Pure pursuit revisited: field testing of autonomous vehicles in urban areas. 2016 IEEE 4th International Conference on Cyber-Physical Systems, Networks and Applications (CPSNA), Nagoya, Japan: IEEE, 2016; pp.7-12.
[14] Abiyev R H, Günsel I S, Akkaya N, Aytac E, Cagman A, Abizada S. Fuzzy control of omnidirectional robot. Procedia Computer Science, 2017; 120: 608-616.

[15] Pandey A, Sonkar R K, Pandey K K, Parhi D R. Path planning navigation of mobile robot with obstacles avoidance using fuzzy logic controller. 2014 IEEE 8th International Conference on Intelligent Systems and Control (ISCO), Coimbatore, India: IEEE, 2014; pp.39-41.

[16] Chen J W, Zhu H, Zhang L, Sun Y. Research on fuzzy control of path tracking for underwater vehicle based on genetic algorithm optimization. Ocean Engineering, 2018; 156: 217-223.

[17] Hwang C L, Yang C C, Hung J Y. Path tracking of an autonomous ground vehicle with different payloads by hierarchical improved fuzzy dynamic sliding-mode control. IEEE Transactions on Fuzzy Systems, 2018; 26(2): 899-914.

[18] Kárník J, Jakub S. Summary of available indoor location techniques. International Federation of Automatic Control, 2016; 49(25): 311-317.

[19] Cotera P, Velazquez M, Cruz D, Medina L, Bandala M. Indoor robot positioning using an enhanced trilateration algorithm. International Journal of Advanced Robotic Systems, 2016; 13(3): $110 . \quad$ doi: $10.5772 / 63246$.

[20] Makki A, Siddig A, Saad M, Cavallaro J R, Bleakley C J. Indoor localization using time differences of arrival. IEEE Transactions on Instrumentation and Measurement, 2015; 65(3): 1-10.

[21] Schreiber R, Bajer J. Time difference measurement algorithm for TDOA positioning system using RTL-SDR. 2017 International Conference on Military Technologies (ICMT), Brno, Czech Republic: IEEE, 2017; pp.608-612. 